\title{
Thorough QT/QTc in a Dish: An In Vitro Human Model That Accurately Predicts Clinical Concentration-QTc Relationships
}

\author{
Alexander D. Blanchette, BS ${ }^{\# 1}$, Fabian A. Grimm, PhD $^{\# 1}$, Chimedullam Dalaijamts, PhD ${ }^{1}$, \\ Nan-Hung Hsieh, PhD ${ }^{1}$, Kyle Ferguson, MS ${ }^{1}$, Yu-Syuan Luo, PhD ${ }^{1}$, Ivan Rusyn, MD PhD ${ }^{1}$, \\ and Weihsueh A. Chiu, PhD $^{1}$ \\ ${ }^{1}$ Department of Veterinary Integrative Biosciences, Texas A\&M University, College Station, TX \\ \# These authors contributed equally to this work.
}

\begin{abstract}
"Thorough QT/QTc" (TQT) studies are cornerstones of clinical cardiovascular safety assessment. However, TQT studies are resource intensive, and preclinical models predictive of the threshold of regulatory concern are lacking. We hypothesized that an in vitro model using iPSC-derived cardiomyocytes from a diverse sample of human subjects can serve as a "TQT study in a dish." For 10 positive and 3 negative control drugs, in vitro concentration-QTc, computed using a population Bayesian model, accurately predicted known in vivo concentration-QTc. Moreover, predictions of the percent confidence that the regulatory threshold of $10 \mathrm{msec}$ QTc prolongation would be breached were also consistent with in vivo evidence. This "TQT study in a dish," consisting of a population-based iPSC-derived cardiomyocyte model and Bayesian concentrationQTc modeling, has several advantages over existing in vitro platforms, including higher throughput, lower cost, and the ability to accurately predict the in vivo concentration range below the threshold of regulatory concern.
\end{abstract}

\section{Keywords}

Stem Cells; Cardiovascular Risk; QTc Interval; Bayesian; In Vitro; In vitro in vivo correlation

\section{Introduction}

Cardiovascular adverse effects are a significant concern in all phases of drug development (1). To address this concern, multipronged screening strategies that include in silico, in vitro, non-clinical and clinical studies have been implemented in drug development and safety

\footnotetext{
Address correspondence to: Weihsueh A. Chiu, PhD, Department of Veterinary Integrative Biosciences, 4458 TAMU, Texas A\&M University, College Station, TX 77843, (979) 845-4106, fax: (979) 847-8981, wchiu @cvm.tamu.edu. Author Contributions:

A.D.B., I.R., and W.A.C. wrote the manuscript; I.R. and W.A.C. designed the research; F.A.G., A.D.B., K.F., Y.S.L., C.D., and N-H.H. performed the research; A.D.B. and W.A.C. analyzed the data.

Conflict of Interest Statement

The authors declared no competing interests for this work.

Supplemental Information Titles

File 1: Supplemental Material: Model Code, Supplemental Figures, Supplemental Tables

File 2: CSV-format Data file
} 
evaluation $(2,3)$. One of the major cardiovascular safety concerns is the potential of a new drug to prolong the QT interval because it is a significant risk factor for Torsade de Pointes (TdP), a ventricular arrhythmia that in some cases can be lethal (4). The electro-

physiological mechanisms of drug-induced prolonged QT intervals are well established, in humans they are usually caused by the drug's ability to inhibit IKr which is the rapid component of the delayed rectifier potassium current encoded by ether-a-go-go related gene $(h E R G)(5)$. Therefore, drug candidates are routinely evaluated for IKr inhibitory potency using voltage clamp studies of hERG function in vitro in conjunction with in vivo testing in a non-rodent animal model (3). Excellent concordance exists between QT results in humans and non-rodent animals; for example, a systematic review of 23 positive and 17 negative drugs showed that QT interval data derived from relevant non-rodent models has a $90 \%$ chance of predicting QT findings in humans (6). The assessment of the effect on hERG has been shown to be a valuable indicator of a potential long QT liability. Both absolute hERG $\mathrm{IC}_{50}$, and a safety margin between $\mathrm{hERG} \mathrm{IC}_{50}$ and clinical peak free plasma exposure, have similar positive and negative predictive values to those derived from studies comparing a safety margin between QTc effects in dogs and clinical peak free plasma exposure (4).

While preclinical studies have recognized value, ultimately, a clinical "Thorough QT/QTc" (TQT) study is still required, even without preclinical findings indicative of safety concerns. The International Conference on Harmonization (ICH) E14 guideline (7) for the TQT study states that the "threshold level of regulatory concern... is around $5 \mathrm{~ms}$ as evidenced by an upper bound of the 95\% confidence interval around the mean effect on QTc of $10 \mathrm{ms."}$ Overall, incorporation of these preclinical and clinical tests has been widely credited with the reduction in drugs with pro-arrhythmic risk (8), but the high cost of the TQT study, estimated to be 1 to 4 million dollars (9), has led to efforts to develop both clinical and preclinical alternatives. For instance, both ICH and FDA now allow an alternative TQT study that involves concentration-QTc (C-QTc) modeling of QTc data collected in early phase 1 studies (10-15).

Additionally, human stem cell-derived in vitro models have also made considerable inroads into routine testing for cardiovascular liabilities (16). The ability to generate cells from multiple donors that replicate patient-specific congenital (17) and disease-specific (18) phenotypes is an exciting development that promises to enable personalized drug safety evaluation, an approach that has recently been tested in dozens of human induced pluripotent stem cell (iPSC)-derived cardiomyocytes $(18,19)$. iPSC-based cardiomyocytes are known to be useful in identifying both congenital and drug-induced cardiotoxicity hazards $(17,20$, $21)$, particularly at the individual patient level $(22,23)$. However, even within the Comprehensive in vitro Proarrhythmia Assay (CiPA) initiative - a global effort among regulators, industry, and academia to develop a mechanistic, model-informed approach to cardiac safety that includes iPSC-derived cardiomyocytes as a key component - clinical testing for QT prolongation through in vivo ECG monitoring in Phase I clinical trials remains a key component (24).

The success of applying C-QTc modeling to clinical QT prolongation, along with the potential for iPSC-derived cardiomyocytes to recapitulate in vivo effects, suggests that there may be potential in combining the two approaches in a preclinical setting. We have 
previously shown that iPSC-derived cardiomyocytes from a moderate-sized sample of healthy subjects $(n=27)$ are a highly reproducible in vitro population model (19). Notably, this sample size is greater than those recommended to control false negatives in C-QTc modeling-based TQT studies (14). We therefore hypothesized that the C-QTc modeling of iPSC-derived cardiomyocytes from a diverse sample of human subjects can serve as a "TQT study in a dish." To test this hypothesis, we first investigate whether a population-based in vitro model in combination with in silico PD modeling can predict the clinical C-QTc relationship (Figure 1A). Then, we demonstrate clinical translation, determining the range of clinical concentrations satisfying the TQT study regulatory threshold (Figure 1B).

\section{Results}

\section{Determination of Free Fraction in vivo and in vitro:}

Measured free fractions in plasma $\left(f_{\text {plasma }}\right)$ and cardiomyocyte media $\left(f_{\text {media }}\right)$ are summarized in Table 1. The percentage unbound ranged from $89 \%$ to $108 \%$ in PBS buffer, showing full equilibration between sample and control chambers. For plasma, measured $f_{\text {plasma }}$ correlated well with reported literature values, although in many cases the measured values were higher. In all cases, $f_{\text {media }}>f_{\text {plasma }}$, as expected due to the media containing less protein. Measured media values correlated well with values calculated using the Armitage et al. (25) model, although for citalopram and lamotrigine, measured $f_{\text {media }}>100 \%$. For in vivo-in vitro comparison, measured values were used up to a maximum of $100 \%$ free.

\section{$\mathrm{Ca}^{2+}$ Flux Assay:}

The 27 donors exhibited reproducible inter-individual variation both at baseline (19) and with treatment with all 10 positive QT drugs. However, all 10 positive QT drugs exhibited visible increases in the decay-rise ratio at one or more tested concentrations in multiple donors. Additionally, in all positive controls, formation of a "notch" (where the $\mathrm{Ca}^{2+}$ flux partially declines, then "plateaus" for a period before completely returning to baseline, see Figure 1A) was observed in at least 3 of the 27 donors. No "notch" formation occurred for any of the 3 negative QT drugs in any donor. Presence of a "notch" is considered an indicator of arrhythmic beating (21). The data are available as Supplemental Materials.

\section{Bayesian Population Modeling of in vitro Data:}

Population concentration-response modeling of the decay-rise ratio successfully converged for all 13 drugs ( $\widehat{R} \leq 1.05$ for all parameters). Modeling results accurately fit the experimental in vitro data, with typical residual standard errors (RSE) of less than $20 \%$ (Figure 2A) and the data are well within 95\% credible intervals (CI) (Figure 2B for disopyramide; all drugs are shown in Figures S1-S13). The poorest model fit was for cisapride, with a $\log _{10}$-transformed RSE of 0.17 and $\mathrm{R}^{2}$ of 0.82 . Because cells from many donors became quiescent above the lowest tested cisapride concentration, data were often only available for a single treatment at $0.1 \mu \mathrm{M}$. For citalopram, the RSE was 0.11 and $\mathrm{R}^{2}$ was 0.68 , but these values were driven by a single outlier point. Other positive controls had $\mathrm{RSE}<0.1$ and $\mathrm{R}^{2}>0.85$. Vehicle and negative drug controls also all had $\operatorname{RSE}<0.1$, but lower $\mathrm{R}^{2}$ due to the lack of effect at most tested concentrations. 


\section{In vivo-in vitro Comparisons:}

The in vitro and in vivo free concentration-response relationships were highly concordant across all positive controls, with the $95 \% \mathrm{CI}$ of the in vitro prediction for the population median almost always overlapping with the in vivo concentration-response (Figure 3A for disopyramide; all drugs in Figures S14-S26). Importantly, the 95\% CI prediction for the "random" donor in vitro is wide, indicating that any single donor may be much more or less sensitive pharmacodynamically than in vivo populations studied.

The positive controls dofetilide and cisapride have clinical $\mathrm{C}_{\max }$ values below the tested range of concentrations in vitro. However, even in these cases, the in vitro model predictions at these lower concentrations remain consistent with the in vivo responses (see Figures S14 and S17). For negative controls, some donors exhibited increases in decay-rise ratio at the highest tested concentration. In the cases of cabazitaxel and mifepristone, the concentrations were well above ( $>20$-fold for $\mathrm{EC}_{01}$ ) clinical $\mathrm{C}_{\max }$, but for lamotrigine, the difference was smaller ( $<2$-fold for lower confidence bound on $\mathrm{EC}_{01}$ ) (Figures S24-S26). Thus, consistent with the in vivo studies, within the clinically relevant range of concentrations, in vitro predictions showed little or no effect.

The degree of in vitro-in vivo concordance was quantified using predictions at specific points on the concentration-response curve: percent effect at the clinical $\mathrm{C}_{\max }\left(\mathrm{EC}_{\max }\right)$ and the effective concentrations for a $1 \%, 5 \%$, and $10 \%$ change $\left(\mathrm{EC}_{01}, \mathrm{EC}_{05}\right.$, and $\left.\mathrm{EC}_{10}\right)($ Table 2). As illustrated in Figures 3C-D and Figures S27-S29, the population median predictions were consistently more accurate than the predictions of any individual random donor.

\section{Clinical Translation to a TQT Study:}

Clinical translation of in vitro C-QTc modeling results involves determining the probability that clinical $\Delta \mathrm{QTc}\left(x_{\text {plasma }}\right)>10 \mathrm{~ms}$; a probability of $<5 \%$ indicates with $95 \%$ confidence that the drug at $x_{\text {plasma }}$ increases QTc by no more than $10 \mathrm{~ms}$. This translation involves scaling on both the concentration axis from in vitro media to in vivo plasma as well as the response axis from percent change in decay-rise ratio in vitro to the ms change in QTc in vivo (see Methods, Figure 1B). The results are shown in Figure 4. For all the positive controls except moxifloxacin, our "TQT in a dish" clearly predicts that the regulatory threshold would be breached at the clinical $\mathrm{C}_{\max }$. For moxifloxacin, the $\mathrm{C}_{\max }$ is barely within the regulatory threshold for the population median prediction, but fails for the individual donor predictions. For the negative controls, both cabaitaxel and mifepristone clearly satisfy the regulatory threshold. However, for lamotrigine, similar to moxifloxacin, the $\mathrm{C}_{\max }$ is barely within the regulatory threshold for the population median prediction, but fails for individual donor predictions.

\section{Discussion}

Our study hypothesized that C-QTc modeling using population of iPSC-derived cardiomyocytes could serve as an in vitro alternative to the TQT study, incorporating six key, novel components: 
1. First, we hypothesized that a population of iPSCs, rather than a single donor, would improve predictive accuracy. This hypothesis was motivated by studies showing that congenital susceptibilities are recapitulated in iPSC-derived cardiomyocytes $(17,22,23,26,27)$ as well as previous studies showing good correlation but poor quantitative accuracy between in vitro and in vivo effective concentrations using a single donor (28). We found that by averaging over the unique susceptibilities of individual donors, our population-based predictions were more accurate than those based on any single donor.

2. Second, we posited that the in vitro decay-rise ratio (time from peak to baseline divided by time from baseline to peak) of calcium flux could be a high throughput, easily measured surrogate for the in vivo QTc interval. The decayrise ratio exhibited behaviors directly analogous to QTc: (i) similar to how RR is used to standardize QT intervals, the decay-rise ratio appropriately adjusted for differences in baseline beat rate, and (ii) similar to QTc being a precursor for more severe arrhythmias, increases in the decay-rise ratio preceded in vitro arrhythmias such as "notch" formation.

3. Third, we fit concentration-response data using a Bayesian population model to enable both population and individual-level predictions (Figure 2), enabling in vivo and in vitro comparisons of the full concentration-response relationships both rather than at only specific concentrations (Figures 3A-B).

4. Fourth, we hypothesized that the percent change from baseline in the in vitro decay-rise ratio would predict the percent change from baseline in the in vivo QTc interval, which our results demonstrated to be accurate, particularly at lower effect sizes most relevant to regulatory concerns (Figure 3C).

5. Fifth, we demonstrated how our model could be clinically translated to predict the regulatory threshold of 95\% confidence of QTc-prolongation < $10 \mathrm{msec}$, which is the aim of traditional TQT studies. Our results definitively predicted QTc-prolongation concerns for 9 of the 10 positive controls. For one of the 10 positive controls, moxifloxacin, the results were "borderline," as the populationbased prediction suggested $<5 \%$ probability of a $10 \mathrm{~ms}$ QTc-prolongation, but predictions based on individual donors (e.g., a "random individual") indicated a $>5 \%$ probability. This ambiguity is consistent with the evidence in the literature, as studies indicate that clinical $\mathrm{C}_{\max }$ values result in QTc-prolongation near the $10 \mathrm{~ms}$ threshold $(29,30)$. For negative controls, our results definitively predicted lack of QTc-prolongation concerns in 2 of the 3 compounds. Lamotrigine was "borderline," like moxifloxacin, showing $<5 \%$ probability for the population, but $>5 \%$ probability for individual random donors. This ambiguity is also corroborated by clinical reports: although the TQT study of lamotrigine in healthy volunteers showed no effect on QTc (hence its classification as a "negative" control), several case reports and series have reported QTc prolongation with lamotrigine in susceptible patients $(31,32)$.

6. Finally, unlike most assays investigating arrhythmias in iPSC-derived cardiomyocytes, our experimental protocol is suited to rapid high throughput 
screening in a 384-well format, with both cell lines, equipment and reagents, and data analysis software available commercially or as open source software. For instance, our protocol contrasts with the technologies investigated in the multisite validation study as part of the CiPA initiative (i.e., multi-electrode array and voltage-sensing dye), which are both more expensive and much lower throughput.

While encouraging, our study has several limitations. First is that we only tested 10 positive and 3 negative drugs, numbers limited by openly available in vivo PK-PD models and data for QTc prolongation in the literature. With the recent emphasis on C-QTc modeling of early phase clinical data, additional data are becoming available. Second, while our main goal was to demonstrate that this model can serve as an in vitro version of the traditional TQT study a "TQT in a dish" - it remains to be established whether our approach is sufficiently accurate for other indicators of cardiovascular toxicity, including TdP. Thus, whether iPSCs can play an expanded role beyond screening for QTc prolongation remains to be determined. Third, expanding the number of donors for these studies is desirable, particularly for the purpose of investigating population variation. Additional cells from "normal" donors are available from various academic laboratories and may be used to increase the population size; however, their availability to any future user may be more constrained as compared to cells available and quality-controlled by a commercial vendor.

In summary, our results indicate that it is possible to predict quantitatively the in vivo C-QTc relationship in the clinically relevant range of concentrations through use of an in vitro-in silico model consisting of protein binding measurements, population of iPSC-derived cardiomyoctes, functional parameters assayed by $\mathrm{Ca}^{2+}$ flux, and population Bayesian concentration-response modeling. Moreover, these results can be clinically translated to TQT studies - a "TQT study in a dish" - providing, to the best of our knowledge, the first prediction of the concentration range below the regulatory threshold of concern for QTc prolongation. Our approach has the further advantage that it is a readily accessible to "offthe-shelf" high throughput screening, with all methods and materials either commercially available or open source and amenable to standard 384-well plate-based screening. Thus, in addition to drugs, other xenobiotic exposures such as consumer product chemicals and environmental pollutants can be rapidly and accurately assessed for cardiotoxicity risks. Overall, we believe that expanded use of populations of iPSC-derived in combination with concentration-response modeling represents a translational opportunity that will enable more accurate and successful management of cardiotoxicity risks from drugs and other xenobiotics.

\section{Methods}

\section{Chemicals and Biologicals:}

Cardiomyocyte plating and maintenance media were obtained from FUJIFILM Cellular Dynamics (Madison, WI). Tissue culture grade dimethyl sulfoxide (DMSO, CAS: 67-68-5) was from Santa Cruz Biotechnology (Dallas, TX). Trypan Blue (0.4\%) and penicillin/ streptomycin $(50 \mathrm{mg} / \mathrm{ml})$ were from Life Technologies (Grand Island, NY). Drugs are listed in Table 1 with CAS, catalog number, and source. Phosphate buffer saline (PBS), LC/MS 
grade acetonitrile, LC/MS grade water with $0.1 \%$ formic acid were from Fisher Scientific (Waltham, MA). Human plasma was from Bioreclamation (Westbury, NY) and all donors of plasma tested negative for viral antigens.

\section{Determination of free fraction in vivo and in vitro:}

Protein binding was determined for each chemical utilizing the rapid equilibrium dialysis (RED) assay (catalog no. 90006, Pierce Biotechnology, Rockford, IL) as detailed in (33). Non-specific binding of each chemical was further assessed by incorporating protein free equilibrium controls (PBS buffer controls) in sample chambers. DMSO chemical stock solutions $(10 \mathrm{mM})$ were diluted 100 fold with water to prepare working stock solutions. Twenty microliters of working stock solution was spiked in $180 \mu \mathrm{L}$ of human plasma, cardiomyocyte media, or PBS buffer to the final concentration of $10 \mu \mathrm{M}$ in sample chambers. Final DMSO concentration did not exceed $0.1 \%$. All samples were spiked with 10 $\mu \mathrm{L}$ of $1 \mu \mathrm{M}$ ranolazine (CAS: 95635-55-5, CN: A8510, APExBIO, Houston, TX) as internal standard. The HPLC-MS/MS analysis was performed using Agilent 1260 Infinity II Quaternary system (Waldbronn, Germany) coupled with an Agilent 6470 triple quadrupole mass spectrometer (Santa Clara, CA). Chromatography and the single reaction monitoring (SRM) parameters for 13 drugs are detailed in Table S1. Free fraction was calculated by comparing the response ratios to the concentration of internal standard (IS) within both chambers, sample and buffer, as the following formula: $f_{\text {sample }}=\%$ Free $=($ Chemical response/IS response $)_{\text {buffer }} \div(\text { Chemical response/IS response })_{\text {sample, }}$, where "sample" $=$ "plasma" or "media." For plasma, results were compared to $f_{\text {plasma }}$ reported in the literature (see Table S2); for cardiomyocyte media, results were compared to $f_{\text {media }}$ calculated using the mass-balance model of Armitage et al. (25) (see Tables S3-S4).

\section{iPSC-derived Cardiomyocytes, Cell Culture, and $\mathrm{Ca}^{2+}$ Flux Assay:}

In vitro testing of cardiomyocytes was performed as previously reported (19). The donor population consisted of 12 females and 15 males, of which $85 \%$ were of Caucasian $(n=23)$ and $15 \%$ African American $(n=4)$ ancestry. Catalogue numbers and demographic information on each cell line were previously reported (19). iPSC cardiomyocytes were cultured under identical conditions in multiple batches using an established protocol (34, 35). The $\mathrm{Ca}^{2+}$ flux assay was used to evaluate functional performance of cardiomyocytes $(34,35)$. Example traces for disopyramide treatment and controls are shown in Figure 1A. $\mathrm{Ca}^{2+}$ flux data were analyzed in $\mathrm{R}$ studio (version 1.0.136, with $\mathrm{R}$ version 3.3.2) to estimate relevant functional parameters, as previously described (19). QTc prolongation is indicated by an increase in the decay-rise ratio, as this reflects a delay in the ability to repolarize the action potential and start another beat. The use of this ratio adjusts for the fact that slower beating alone increases the decay time, but not the decay-rise ratio, and is similar to the use of RR in the QTc to adjust for heart rate when measuring the QT interval. Additionally, the analysis identifies traces where a "notch" is present in which the $\mathrm{Ca}^{2+}$ flux partially declines, then "plateaus" for a period before completely returning to baseline (see Figure 1A).

Concentrations above which the "notch" first appears were not included in modeling of the decay-rise ratio because the phenotype then progresses to more severe arrhythmias. 


\section{Bayesian Population C-QTc Modeling of in vitro Data:}

For each drug, concentration-response data for the decay/rise ratio was fit using a hierarchical Bayesian (random-effects) Hill model as previously described (36). The Hill model at the individual donor level was parameterized as $y=y_{0} \times\left(1+\left(x / x_{0}\right)^{n} /(1+(x /\right.$ $\left.\left.\left.x_{0}\right)^{n} / E_{\max }\right)\right)+\varepsilon$, so that $y_{0}$ is value without treatment, $E_{\max }$ is the maximum fractional change from baseline, $x_{0}$ is the concentration at half of the maximal response, $x$ is the treatment nominal concentration, $n$ the Hill coefficient, and $\varepsilon$ is the residual error. The four individual level parameters $y_{0}, E_{\max }, x_{0}$, and $n$, all being strictly positive, were natural-log transformed, with transformed parameters assumed to have normal random effects with population mean and variance hyperparameters. Based on previous experience with high throughput in vitro data $(36,37)$, a robust error model was needed due to the presence of outliers, so the error $\varepsilon$ was assumed to follow a student's t-distribution $\mathrm{t}_{5}(0, \sigma)$ with five degrees of freedom, centered on 0 with scale parameter $\sigma$. Minimally-informative prior distributions were used (see Supplemental Materials).

Posterior distribution sampling was conducted using the Markov Chain Monte Carlo algorithm implemented into the STAN software package (version 2.17.3) (38), interfaced with $R$ (version 3.3.2). Each simulation consisted of 4 chains with 4000 iterations per chemical, with the first 2000 being "warm-up" samplings, which were subsequently discarded. Convergence was assessed by comparing both inter-chain and intra-chain variability for each parameter, with the potential scale reduction factor $\widehat{R} \unlhd .2$ considered converged (39). Model code is included in Supplemental Materials.

Posterior predictions (median and 95\% credible interval) for concentration-response were made using (i) the overall population median; (ii) the standard donor (iCell cardiomyocyte, donor 1434); and (iii) a "random individual" donor drawn from the population. Specifically, for (i) and (ii), 8000 posterior samples for the population median and for the standard donor were used directly; for (iii) 8000 random populations of 100 individuals each were generated using posterior samples of the population mean and variance, and then combined to generate the uncertainty distribution for a "random individual" donor.

\section{In vivo C-QTc Relationships from the Literature:}

The 13 drugs tested were selected based on the availability (at the time of study design) of in vivo PK-PD models that estimated the in vivo concentration-response relationship between serum concentration and change in QTc. For each drug, the C-QTc portion of the PK-PD model was extracted from the corresponding publication (see Table S5). All concentration units were converted to free ("unbound") concentrations based on protein binding measurements described above. Studies differed in reported baseline QTc values (368 to 450 $\mathrm{ms}$ ), so percent change was used as the standard effect measure to better compare across studies, as well as to enable comparison with results from modeling of in vitro data (see In Vivo-In Vitro Comparison, below). As a benchmark, for a baseline QTc of 421.5 ms [mean of NHANES III as previously reported (40)], 1\%, 5\%, and 10\% change correspond to 4.2, 20.1, and $42.2 \mathrm{~ms}$ QTc prolongation. Predictions were restricted to values below the $\mathrm{C}_{\max }$, so that the C-QTc models would not be extrapolated beyond the observed range. 


\section{In Vivo-In Vitro Comparison:}

For the purposes of comparing literature PK-PD in vivo predictions to the in vitro modelling predictions, common metrics for both concentration and have to be established. For concentration, the measured free concentration in either plasma or maintenance media was used, as this represents the amount bioavailable to cells. For response, the percent change from baseline was used, specifically: in vivo, the percent change in QTc; in vitro, the percent change in the decay-rise ratio. Using these metrics, comparisons between in vivo and in vitro predictions were visualized in three ways: the overall free C-QTc relationship, the percent change in QTc at the clinical $\mathrm{C}_{\max }\left(\mathrm{EC}_{\max }\right)$, and the effective concentrations for changes in QTc of $1 \%\left(\mathrm{EC}_{01}\right), 5 \%\left(\mathrm{EC}_{05}\right)$, and $10 \%\left(\mathrm{EC}_{10}\right)$.

\section{Clinical Translation to a TQT Study:}

Under our modeling approach, results of in vitro C-QTc modeling can be clinically translated to the determination of the regulatory threshold for QTc prolongation. In general, the fractional change as a function of in vitro concentration $\mathrm{x}$ predicted from the in vitro model is multiplied by the baseline QTc $=\mathrm{QTc}_{0}$ to predict the QTc change: $\Delta \mathrm{QTc}(x)=\mathrm{QTc}_{0}$ $\times\left(x / x_{0}\right)^{n} /\left(1+\left(x / x_{0}\right)^{n} / E_{\max }\right)$. First, the in vitro concentration is scaled to plasma concentration by $x_{\text {plasma }}=x \times f_{\text {media }} / f_{\text {plasma }}$, For the regulatory threshold $\Delta \mathrm{QTc}^{*}=10 \mathrm{~ms}$, and taking the posterior samples of the model parameters, we determine the probability of satisfying the regulatory threshold $\mathrm{P}\left(\Delta \mathrm{QTc}\left(x_{\text {plasma }}\right)>\Delta \mathrm{QTC} *\right)$ as a function of $x_{\text {plasma }}$. Plasma concentrations for which $\mathrm{P}<5 \%$ satisfy the regulatory threshold. Because it is the population level that is relevant for the clinical TQT study, we use the population median values for the model parameters $x_{0}, n$, and $E_{\max }$, and QTc $\mathrm{C}_{0}=421.5 \mathrm{~ms}$, the mean of male and female QTc (Fridericia) from NHANES III(40). For comparison, we also calculated the regulatory threshold using only the standard donor (\#1434) with $\mathrm{QTc}_{0}=426 \mathrm{~ms}$ (the mean for females(40)), as well as for a "random individual donor" with a randomly sampled QTc $\mathrm{C}_{0}$ with mean $421.5 \mathrm{~ms}$ and standard deviation $22.5 \mathrm{~ms}$ (40).

\section{Supplementary Material}

Refer to Web version on PubMed Central for supplementary material.

\section{Acknowledgments}

Funding

This work was funded, in part, by grants from the National Institutes of Health (P42 ES027704 and T32 ES026568) and a cooperative agreement with the United States Environmental Protection Agency (STAR RD83580201). Fabian Grimm was the recipient of the Society of Toxicology Colgate-Palmolive and Society of Toxicology Syngenta Fellowship Awards. The views expressed in this manuscript do not reflect those of the funding agencies. The use of specific commercial products in this work does not constitute endorsement by the funding agencies.

\section{REFERENCES}

(1). Laverty $\mathrm{H}$ et al. How can we improve our understanding of cardiovascular safety liabilities to develop safer medicines? British journal of pharmacology 163, 675-93 (2011). [PubMed: 21306581]

(2). Arrigoni C \& Crivori P Assessment of QT liabilities in drug development. Cell Biol Toxicol 23, 113 (2007). [PubMed: 17013551] 
(3). Wallis R et al. CiPA challenges and opportunities from a non-clinical, clinical and regulatory perspectives. An overview of the safety pharmacology scientific discussion. J Pharmacol Toxicol Methods, (2018).

(4). Pollard CE et al. An Analysis of the Relationship Between Preclinical and Clinical QT IntervalRelated Data. Toxicol Sci 159, 94-101 (2017). [PubMed: 28903488]

(5). Redfern WS et al. Relationships between preclinical cardiac electrophysiology, clinical QT interval prolongation and torsade de pointes for a broad range of drugs: evidence for a provisional safety margin in drug development. Cardiovasc Res 58, 32-45 (2003). [PubMed: 12667944]

(6). Vargas HM et al. Evaluation of drug-induced QT interval prolongation in animal and human studies: a literature review of concordance. British journal of pharmacology 172, 4002-11 (2015). [PubMed: 26031452]

(7). International Conference on Harmonization. The Clinical Evaluation of QT/QTC Interval Prolongation and Proarrythmic Potential for Non-Antiarrythmic Drugs E14. (INTERNATIONAL CONFERENCE ON HARMONISATION OF TECHNICAL REQUIREMENTS FOR REGISTRATION OF PHARMACEUTICALS FOR HUMAN USE, Geneva, Switzerland, 2005).

(8). Park E, Willard J, Bi D, Fiszman M, Kozeli D \& Koerner J The impact of drug-related QT prolongation on FDA regulatory decisions. Int J Cardiol 168, 4975-6 (2013). [PubMed: 23920061]

(9). Bouvy JC, Koopmanschap MA, Shah RR \& Schellekens H The cost-effectiveness of drug regulation: the example of thorough QT/QTc studies. Clin Pharmacol Ther 91, 281-8 (2012). [PubMed: 22205197]

(10). E14 Implementation Working Group. ICH E14 Guideline: The Clinical Evaluation of QT/QTc Interval Prolongation and Proarrhythmic Potential for Non-Antiarrhythmic Drugs Questions \& Answers (R3) (International Council for Harmonisation of Technical Requirements for Pharmaceuticals for Human Use, Geneva, Switzerland, 2015).

(11). US FDA. E14 Clinical Evaluation of QT/QTc Interval Prolongation and Proarrhythmic Potential for Non-Antiarrhythmic Drugs — Questions and Answers (R3) Guidance for Industry. (ed. Center for Drug Evaluation and Research (CDER)) (U.S. Department of Health and Human Services, Food and Drug Administration, Silver Spring, MD, 2017).

(12). Shah RR, Maison-Blanche P, Robert P, Denis E \& Duvauchelle T Can an early phase clinical pharmacology study replace a thorough QT study? Experience with a novel H3-receptor antagonist/inverse agonist. Eur J Clin Pharmacol 72, 533-43 (2016). [PubMed: 26879827]

(13). Ferber G, Sun Y, Darpo B, Garnett C \& Liu J Study Design Parameters Affecting Exposure Response Analysis of QT Data: Results From Simulation Studies. J Clin Pharmacol 58, 674-85 (2018). [PubMed: 29420838]

(14). Garnett C et al. Scientific white paper on concentration-QTc modeling. J Pharmacokinet Pharmacodyn 45, 383-97 (2018). [PubMed: 29209907]

(15). Lu J, Li J, Helmlinger G \& Al-Huniti N Assessing QT/QTc interval prolongation with concentration-QT modeling for Phase I studies: impact of computational platforms, model structures and confidence interval calculation methods. J Pharmacokinet Pharmacodyn 45, 46982 (2018). [PubMed: 29556866]

(16). Takasuna $\mathrm{K}$ et al. Comprehensive in vitro cardiac safety assessment using human stem cell technology: Overview of CSAHi HEART initiative. J Pharmacol Toxicol Methods 83, 42-54 (2017). [PubMed: 27646297]

(17). Yang C et al. Concise Review: Cardiac Disease Modeling Using Induced Pluripotent Stem Cells. Stem Cells 33, 2643-51 (2015). [PubMed: 26033645]

(18). Sharma A et al. High-throughput screening of tyrosine kinase inhibitor cardiotoxicity with human induced pluripotent stem cells. Sci Transl Med 9, pii: eaaf2584 (2017). [PubMed: 28202772]

(19). Grimm FA et al. A human population-based organotypic in vitro model for cardiotoxicity screening. ALTEX, (2018).

(20). Kolaja K Stem cells and stem cell-derived tissues and their use in safety assessment. The Journal of biological chemistry 289, 4555-61 (2014). [PubMed: 24362027] 
(21). Blinova K et al. Comprehensive Translational Assessment of Human-Induced Pluripotent Stem Cell Derived Cardiomyocytes for Evaluating Drug-Induced Arrhythmias. Toxicol Sci 155, 234 47 (2017). [PubMed: 27701120]

(22). Chanana AM, Rhee JW \& Wu JC Human-induced pluripotent stem cell approaches to model inborn and acquired metabolic heart diseases. Curr Opin Cardiol 31, 266-74 (2016). [PubMed: 27022891]

(23). Magdy T, Schuldt AJT, Wu JC, Bernstein D \& Burridge PW Human Induced Pluripotent Stem Cell (hiPSC)-Derived Cells to Assess Drug Cardiotoxicity: Opportunities and Problems. Annu Rev Pharmacol Toxicol 58, 83-103 (2018). [PubMed: 28992430]

(24). Vicente J et al. Mechanistic Model-Informed Proarrhythmic Risk Assessment of Drugs: Review of the "CiPA" Initiative and Design of a Prospective Clinical Validation Study. Clin Pharmacol Ther 103, 54-66 (2018). [PubMed: 28986934]

(25). Armitage JM, Wania F \& Arnot JA Application of mass balance models and the chemical activity concept to facilitate the use of in vitro toxicity data for risk assessment. Environ Sci Technol 48, 9770-9 (2014). [PubMed: 25014875]

(26). Burridge PW et al. Human induced pluripotent stem cell-derived cardiomyocytes recapitulate the predilection of breast cancer patients to doxorubicin-induced cardiotoxicity. Nat Med 22, 547-56 (2016). [PubMed: 27089514]

(27). Chen IY, Matsa E \& Wu JC Induced pluripotent stem cells: at the heart of cardiovascular precision medicine. Nat Rev Cardiol 13, 333-49 (2016). [PubMed: 27009425]

(28). Pfeiffer ER, Vega R, McDonough PM, Price JH \& Whittaker R Specific prediction of clinical QT prolongation by kinetic image cytometry in human stem cell derived cardiomyocytes. J Pharmacol Toxicol Methods 81, 263-73 (2016). [PubMed: 27095424]

(29). Matsukura $S$ et al. Effects of moxifloxacin on the proarrhythmic surrogate markers in healthy Filipino subjects: Exposure-response modeling using ECG data of thorough QT/QTc study. J Pharmacol Sci 136, 234-41 (2018). [PubMed: 29627227]

(30). Tsikouris JP, Peeters MJ, Cox CD, Meyerrose GE \& Seifert CF Effects of three fluoroquinolones on QT analysis after standard treatment courses. Ann Noninvasive Electrocardiol 11, 52-6 (2006). [PubMed: 16472283]

(31). Chavez P, Casso Dominguez A \& Herzog E Evolving Electrocardiographic Changes in Lamotrigine Overdose: A Case Report and Literature Review. Cardiovasc Toxicol 15, 394-8 (2015). [PubMed: 25448877]

(32). Moore PW, Donovan JW, Burkhart KK \& Haggerty D A case series of patients with lamotrigine toxicity at one center from 2003 to 2012. Clin Toxicol (Phila) 51, 545-9 (2013). [PubMed: 23869656]

(33). Wetmore BA et al. Incorporating population variability and susceptible subpopulations into dosimetry for high-throughput toxicity testing. Toxicol Sci 142, 210-24 (2014). [PubMed: 25145659]

(34). Grimm FA, Iwata Y, Sirenko O, Bittner M \& Rusyn I High-Content Assay Multiplexing for Toxicity Screening in Induced Pluripotent Stem Cell-Derived Cardiomyocytes and Hepatocytes. Assay Drug Dev Technol 13, 529-46 (2015). [PubMed: 26539751]

(35). Sirenko O et al. Multiparameter in vitro assessment of compound effects on cardiomyocyte physiology using iPSC cells. J Biomol Screen 18, 39-53 (2013). [PubMed: 22972846]

(36). Chiu WA, Wright FA \& Rusyn I A tiered, Bayesian approach to estimating of population variability for regulatory decision-making. ALTEX 34, 377-88 (2017). [PubMed: 27960008]

(37). Filer DL, Kothiya P, Setzer RW, Judson RS \& Martin MT tcpl: the ToxCast pipeline for highthroughput screening data. Bioinformatics 33, 618-20 (2017). [PubMed: 27797781]

(38). Carpenter B et al. Stan: A Probabilistic Programming Language. J Stat Softw 76, 1-32 (2017).

(39). Gelman A \& Rubin DB Inference from Iterative Simulation Using Multiple Sequences. Statist Sci, 457-72 (1992).

(40). Benoit SR, Mendelsohn AB, Nourjah P, Staffa JA \& Graham DJ Risk factors for prolonged QTc among US adults: Third National Health and Nutrition Examination Survey. Eur J Cardiovasc Prev Rehabil 12, 363-8 (2005). [PubMed: 16079644] 


\section{Study Highlights}

- What is the current knowledge on the topic?

Thorough QT/QTc (TQT) studies are highly resource-intensive studies that provide critical clinical information for cardiovascular safety assessment. Human iPSC-derived cardiomyocytes can successfully screen drugs for effects on cardiac ion channels, but it is unclear if they can be translated to address regulatory QTc-prolongation safety thresholds.

- What question did this study address?

This study asks whether an in vitro, population-based model of human iPSCderived cardiomyocytes can accurately predict in vivo concentration-QTc relationships and drug concentrations above/below the threshold of regulatory concern.

- What does this study add to our knowledge?

A population-based iPSC-derived cardiomyocyte model, combined with Bayesian concentration-QTc modeling, can accurately predict in vivo concentration-QTc and regulatory thresholds for 10 positive and 3 negative control drugs.

- How might this change clinical pharmacology or translational science?

By providing an accurate prediction for the range of in vivo concentrations below the threshold regulatory, this "TQT in a dish" model can inform at the preclinical stage whether proarrhythmic liabilities can be managed relative to therapeutic benefits, potentially reducing the need for clinical TQT studies. 


\section{A: Model Development and Evaluation}
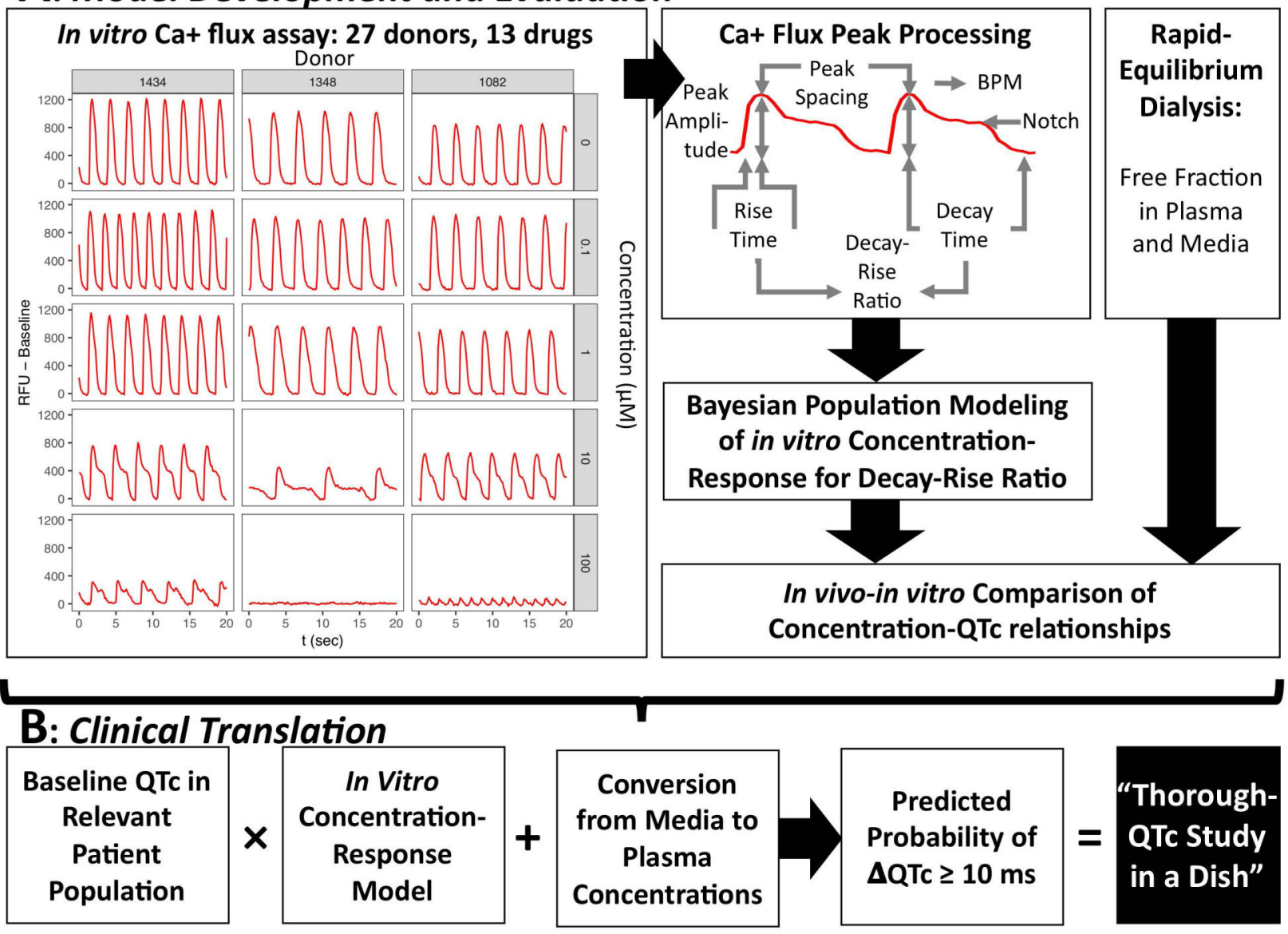

Figure 1. Overview of methods for predicting concentration-response for QTc prolongation and clinical translation to a "Through QTe Study in a Dish."

(A) Model development and evaluation. On the left are representative $\mathrm{Ca} 2+$ flux traces for cells derived from three donors at concentrations of $0,0.1,1,10$, and $100 \mathrm{uM}$ disopyramide. $\mathrm{Ca}+$ flux traces were processed to derive peak parameters, including the decay-rise ratio as the in vitro surrogate for the in vivo QTc. Concentration-response modeling using a Bayesian population approach was then conducted. The resulting concentration-response predictions were then compared to the in vivo concentration-response models, adjusting for differing free fractions in plasma and media. (B) Clinical translation to a Thorough QTc (TQT) Study. The in vitro concentration-response model expressing percent change as a function of media concentration is scaled first to ms change by using the baseline QTc in the relevant patient population, then by converting media concentrations to plasma concentrations. Then, using the posterior distributions of the Bayesian population model, the probability that the change in QTc $(\Delta \mathrm{QTc})$ is greater than or equal to $10 \mathrm{~ms}$ is calculated. This prediction is equivalent to the regulatory threshold determination in a traditional TQT study, which is $95 \%$ percent (one-sided) confidence that the change in QTc is no more than $10 \mathrm{~ms}$. 

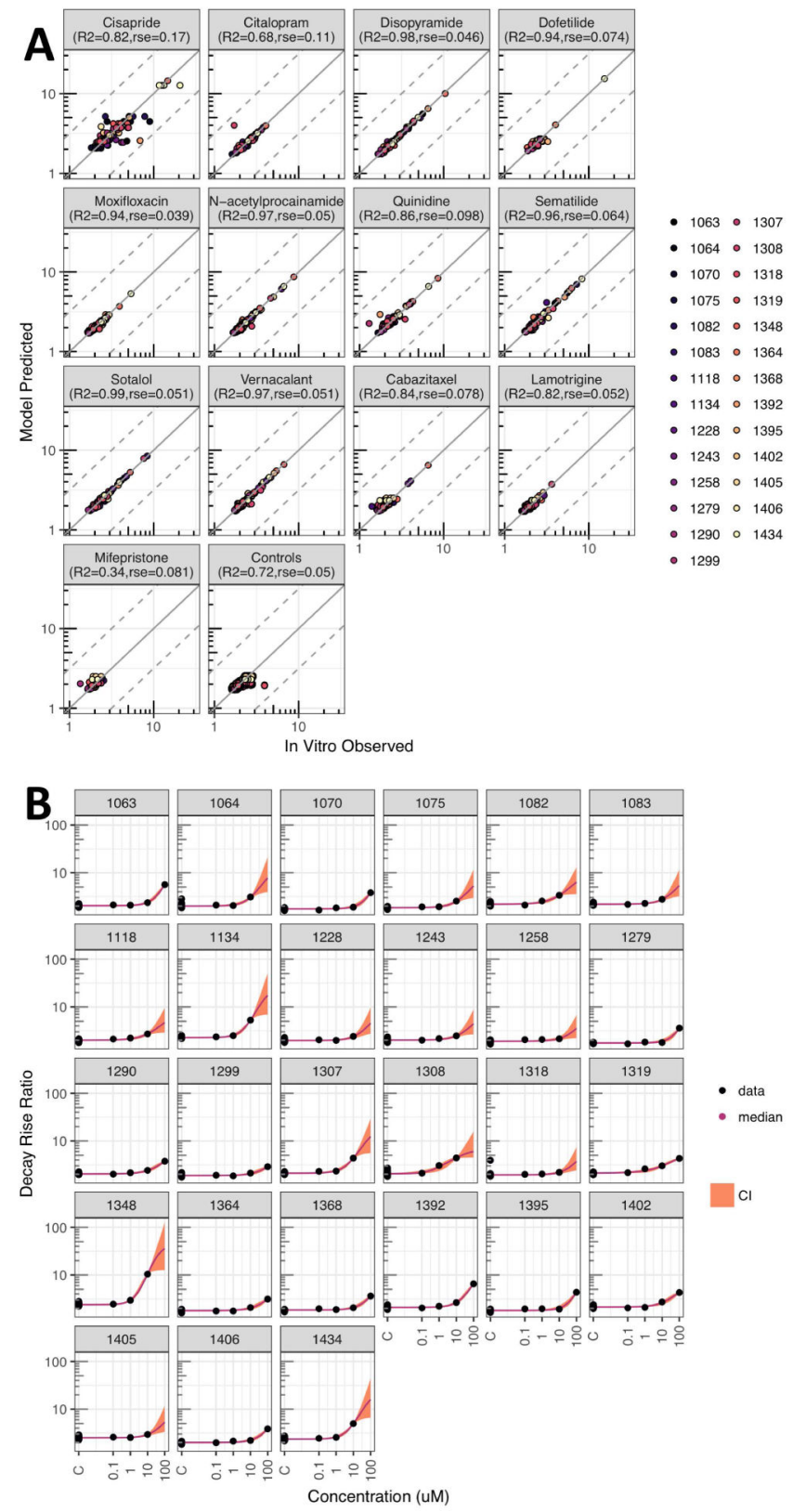

Figure 2. Concentration response modeling results.

(A) Observed in vitro decay-rise ratio data compared to a "random" sample prediction for drugs and controls, each in separate subpanels; different colors denote different individuals. The adjusted $\mathrm{R}^{2}$ and residual standard error (rse) are for predictions vs. data after $\log _{10}$ transformation. (B) Example concentration-response model fit for disopyramide (other in Supplemental Materials) with decay-rise data (black dot); median prediction (colored line) and $95 \%$ CI (colored shading). 

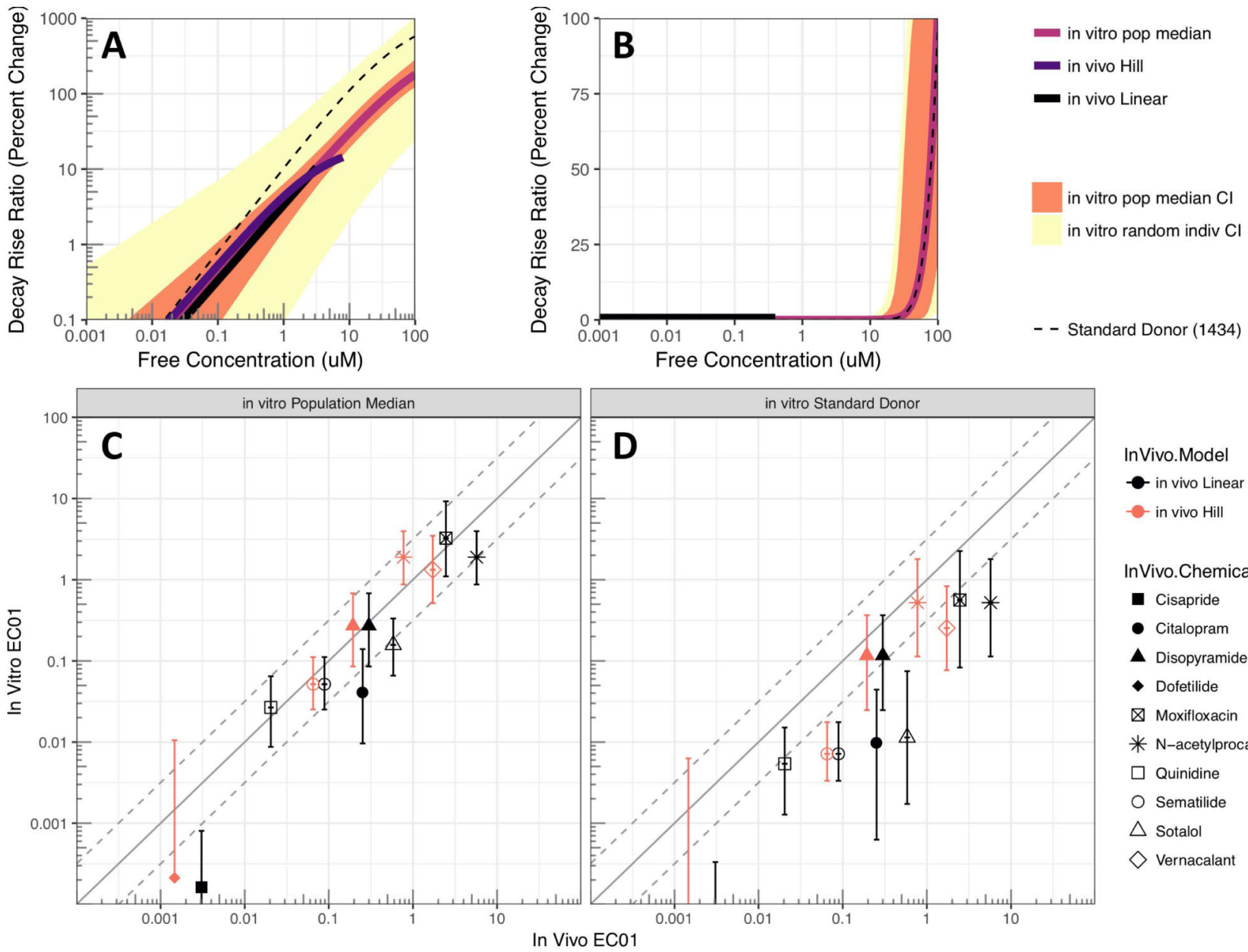

InVivo. Model

- in vivo Hill

InVivo.Chemical.name

- Cisapride

- Citalopram

$\Delta$ Disopyramide

- Dofetilide

$\triangle$ Moxifloxacin

* N-acetylprocainamide

$\square$ Quinidine

○ Sematilide

$\triangle$ Sotalol

$\diamond$ Vernacalant

Figure 3. In vivo-in vitro Comparison.

(A-B) Comparison of concentration-response functions based on in vivo data compared to in vitro data for (A) disopyramide (positive control) and (B) cabazitaxel (negative control). Predictions for in vivo response are shown up to clinical $\mathrm{C}_{\max }$ free; in vitro predictions shown include population median, random individual, and standard donor (1434). Results for other chemicals in Supplemental Materials. (C-D) Comparison of in vivo EC01 with in vitro EC01 based on (C) population median and (D) standard donor (1434). Results for EC05, EC10, and response at $\mathrm{C}_{\max }$ are shown in Supplemental Materials. 

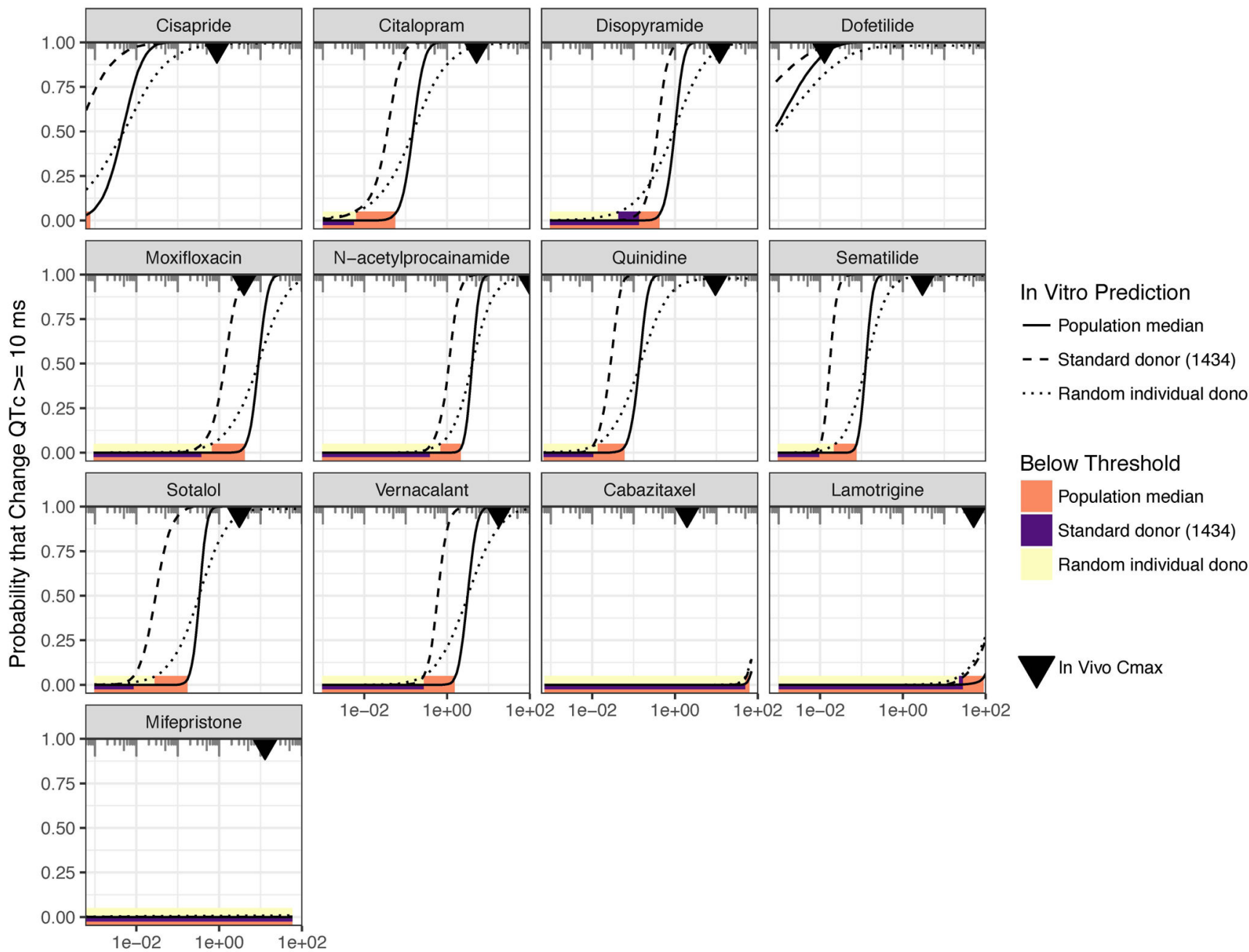

In Vitro Prediction

- Population median

- - Standard donor (1434)

.... Random individual donor

Random individual donor

Plasma Concentration (uM)

Figure 4. Clinical translation to "Thorough-QT in a dish."

In each panel, the probability that QTc is prolonged by $10 \mathrm{~ms}$ or more is plotted against drug plasma concentration. The most direct correspondence to the regulatory threshold for a clinical TQT study is the population median response (solid line). For comparison, the probability is also plotted using only the standard donor (1434) (dashed line) as well as for a "random individual donor," which provide information as to probabilities at the individuallevel rather than population-level. For each probability curve, the solid bars (orange=population median; purple=standard donor; yellow=random individual) represent the plasma concentration range over which the regulatory threshold is met (less than 5\% probability). The inverted triangle represents the clinical $\mathrm{C}_{\max }$ as reported in in vivo PK-PD studies. 
Table 1.

Free fraction in vivo (plasma) and in vitro (cardiomyocyte media)

\begin{tabular}{lcccc}
\hline $\begin{array}{l}\text { Drug (+ or - for QT } \\
\text { prolongation) }\end{array}$ & Plasma & Media & \\
CAS, Catalog Number, Source & Measured & Literature & Measured & Calculated \\
\hline Cisapride (+), CAS: 81098-60-4, CN: C4740, SA & $7 \% \pm 1 \%$ & $2 \%-4 \%$ & $62 \% \pm 1 \%$ & $45 \%-72 \%$ \\
Citalopram hydrobromide (+), CAS: 59729-33-8, CN: C7861, SA & $77 \% \pm 12 \%$ & $20 \%$ & $133 \% \pm 6 \%$ & $57 \%-87 \%$ \\
Disopyramide phosphate (+), CAS: 22059-60-5, CN: D6035, SA & $67 \% \pm 3 \%$ & $25 \%-50 \%$ & $95 \% \pm 5 \%$ & $75 \%-97 \%$ \\
Dofetilide (+), CAS: 115256-11-6, CN: A8417, A & $62 \% \pm 29 \%$ & $17 \%-37 \%$ & $86 \% \pm 7 \%$ & $90 \%-100 \%$ \\
Moxifloxacin hydrochloride (+), CAS: $186826-86-8$, CN: A5323, A & $104 \% \pm 9 \%$ & $30 \%-50 \%$ & $93 \% \pm 2 \%$ & $89 \%-100 \%$ \\
N-acetylprocainamide (+), CAS: 32795-44-1, CN:269476, SA & $98 \% \pm 4 \%$ & $30 \%$ & $96 \% \pm 4 \%$ & $97 \%-99 \%$ \\
Quinidine sulfate (+), CAS: 50-54-4, CN: B7590, A & $37 \% \pm 10 \%$ & $10 \%-77 \%$ & $68 \% \pm 3 \%$ & $55 \%-97 \%$ \\
Sematilide (+), CAS: 101526-83-4, CN: S0323, SA & $85 \% \pm 4 \%$ & $96 \%$ & $96 \% \pm 5 \%$ & $98 \%-99 \%$ \\
Sotalol hydrochloride (+), CAS: 959-24-0, CN: B3341, A & $92 \% \pm 7 \%$ & $100 \%$ & $97 \% \pm 2 \%$ & $99 \%-100 \%$ \\
Vernacalant (+), CAS: 748810-28-8, CN: HY-14183, ME & $79 \% \pm 2 \%$ & $53 \%-63 \%$ & $97 \% \pm 2 \%$ & $26 \%-92 \%$ \\
Cabazitaxel (-), CAS: 183133-96-2, CN: B2157, A & $20 \% \pm 2 \%$ & $5 \%-7 \%$ & $70 \% \pm 9 \%$ & $0.02 \%-92 \%$ \\
Lamotrigine (-), CAS: 84057-84-1, CN: B2249, A & $87 \% \pm 6 \%$ & $45 \%$ & $137 \% \pm 10 \%$ & $86 \%-100 \%$ \\
Mifepristone (-), CAS: 84371-65-3, CN: M8046, SA & $2 \% \pm 1 \%$ & $1 \%-20 \%$ & $58 \% \pm 5 \%$ & $2 \%-9 \%$ \\
\hline
\end{tabular}

Measured values are expressed as mean \pm standard deviation $(\mathrm{n}=3$ ). Sources are Sigma Aldrich, St. Louis, MO (SA), APExBIO, Houston, TX (A), and MedChem Express, Monmouth Junction, NJ (ME). CN=Catalog number. Values reported in the literature (Table S2) or calculated from the Armitage et al. ${ }^{25}$ (2014) model (Tables S3-S4) are expressed as a range. 


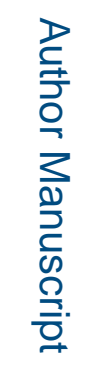

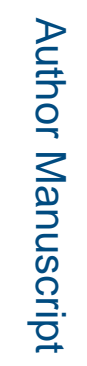

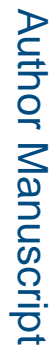

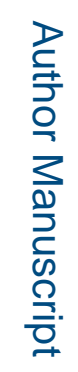

